\title{
The Ratio of the Irredundance Number and the Domination Number for Block-Cactus Graphs
}

\author{
V.E. Zverovich * \\ Department II of Mathematics, RWTH Aachen, Aachen 52056, Germany
}

\begin{abstract}
Let $\gamma(G)$ and $\operatorname{ir}(G)$ denote the domination number and the irredundance number of a graph $G$, respectively. Allan and Laskar [1] and Bollobás and Cockayne [2] proved independently that $\gamma(G)<2 \operatorname{ir}(G)$ for any graph $G$. For a tree $T$, Damaschke [4] obtained the sharper estimation $2 \gamma(T)<3 \operatorname{ir}(T)$. Extending Damaschke's result, Volkmann [11] proved that $2 \gamma(G) \leq 3 \operatorname{ir}(G)$ for any block graph $G$ and for any graph $G$ with cyclomatic number $\mu(G) \leq 2$. Volkmann [11] also conjectured that $5 \gamma(G)<8 \operatorname{ir}(G)$ for any cactus graph. In this article we show that if $G$ is a blockcactus graph having $\pi(G)$ induced cycles of length $2(\bmod 4)$, then $\gamma(G)(5 \pi(G)+4) \leq$ $\operatorname{ir}(G)(8 \pi(G)+6)$. This result implies the inequality $5 \gamma(G)<8 \mathrm{ir}(G)$ for a blockcactus graph $G$, thus proving the above conjecture. J. Graph Theory 29 (1998), 139-149
\end{abstract}

Keywords: graphs, domination number, irredundance number

\section{$1 \quad$ Introduction and Preliminary Results}

All graphs will be finite and undirected, without loops and multiple edges. If $G$ is a graph, $V(G)$ denotes the set of vertices in $G$. The edge set of $G$ is denoted by $E(G)$. Let $N(x)$ denote the neighborhood of a vertex $x$, and let $\langle X\rangle$ denote the subgraph of $G$ induced by $X \subseteq V(G)$. Also let $N(X)=\cup_{x \in X} N(x)$ and $N[X]=N(X) \cup X$. A connected graph with no cut vertex is called a block. A block of a graph $G$ is a subgraph of $G$ which is itself a block and which is maximal with respect to that property. A block $H$ of $G$ is called an end block of $G$ if $H$ has at most one cut vertex of $G$. A graph $G$ is a block graph if every block of $G$ is complete, and $\mathrm{G}$ is a block-cactus graph if every block of $G$ is either a complete graph or a cycle. Block-cactus graphs generalize the known class of cactus graphs. Recall that $G$ is a cactus graph if each edge of $G$ belongs to at most one cycle. If $k(G)$ denotes the number of components of $G$, then $\mu(G)=|E(G)|-|V(G)|+k(G)$ is the cyclomatic number of $G$.

A set $X$ is called a dominating set if $N[X]=V(G)$. The domination number $\gamma(G)$ is the cardinality of a minimum dominating set of $G$. A set $X \subseteq V(G)$ is irredundant if for every vertex $x \in X$,

$$
P_{G}(x, X)=P(x, X)=N[x]-N[X-\{x\}] \neq \emptyset .
$$

\footnotetext{
*On leave from Faculty of Mechanics and Mathematics, Belarus State University, Minsk 220050, Belarus. Supported by the Alexander von Humboldt Foundation.
} 
The minimum cardinality taken over all maximal irredundant sets of $G$ is the irredundance number $\operatorname{ir}(G)$.

It is well known [3] that for any graph $G$,

$$
\operatorname{ir}(G) \leq \gamma(G) .
$$

Allan and Laskar [1] and Bollobás and Cockayne [2] proved independently that $\gamma(G)<$ $2 \operatorname{ir}(G)$ for any graph $G$. For a tree $T$, Damaschke [4] obtained the sharper estimation $2 \gamma(T)<3 \mathrm{ir}(T)$. Extending Damaschke's result, Volkmann [11] proved that $2 \gamma(G) \leq$ $3 \operatorname{ir}(G)$ for any block graph $G$ and for any graph $G$ with cyclomatic number $\mu(G) \leq 2$. Volkmann [11] also posed the following conjecture.

Conjecture 1 (Volkmann [11]) If $G$ is a cactus graph, then

$$
5 \gamma(G)<8 \operatorname{ir}(G) .
$$

In this article, we find the strict ratio of the irredundance and domination numbers for block-cactus graphs having $\pi(G)$ induced cycles of length $2(\bmod 4)$. This result implies the above conjecture. The ratio of related parameters was studied in $[5,7]$. Interesting results for block-cactus graphs can be found in $[6,8,9,10]$.

Proposition 1 (Bollobás and Cockayne [2]) Let I be a maximal irredundant set of the graph $G$. Suppose that the vertex $u$ is not dominated by $I$. Then for some $x \in I$,

a) $P(x, I) \subseteq N(u)$, and

b) for $x_{1}, x_{2} \in P(x, I)$ such that $x_{1} \neq x_{2}$, either $x_{1} x_{2} \in E(G)$ or there exist $y_{1}, y_{2} \in$ $I-\{x\}$ such that $x_{1}$ is adjacent to each vertex of $P\left(y_{1}, I\right)$ and $x_{2}$ is adjacent to each vertex of $P\left(y_{2}, I\right)$.

Let $G$ be a block-cactus graph, $F \subseteq V(G)$ and $W=V(G)-F$. A cycle $C$ in $G$ is called alternating if the sets $F$ and $W$ do not contain edges of $C$. An alternating path is defined analogously.

Lemma 1 Let $G$ be a block-cactus graph and $F \subseteq V(G)$ such that $|N(w) \cap F| \geq 2$ for all $w \in W=V(G)-F$. If $G$ does not contain an alternating cycle $C_{4 k+2}$ as an induced subgraph, then there exists a subset $F^{\prime} \subseteq F$ such that $W \subseteq N\left(F^{\prime}\right)$ and $2\left|F^{\prime}\right| \leq|F|$.

Proof. Without loss of generality we may assume that $\mathrm{G}$ is a connected graph and $W \neq \emptyset$. We prove the lemma by induction on the number of vertices in $G$. The lemma is obvious if $G$ contains few vertices. Suppose that $G$ consists of one block. If $G$ is a complete graph, then the lemma is obvious. Suppose that $G$ is a cycle and consider a maximal alternating path $P$ between $F$ and $W$ in $G$. Let $\langle P\rangle$ be a path. We have

$$
P=f_{1} w_{1} f_{2} w_{2} \ldots f_{t-1} w_{t-1} f_{t}, \quad t \geq 2,
$$

where $f_{i} \in F, 1 \leq i \leq t$, and $w_{i} \in W, 1 \leq i \leq t-1$. The set

$$
D=\left\{f_{2}, f_{4}, f_{6}, \ldots\right\}
$$


dominates the set $P \cap W$ and $|D| \leq t / 2$. The maximal alternating paths in the cycle $G$ are vertex disjoint and hence it is easy to construct the set $F^{\prime}$. Now suppose that $\langle P\rangle$ is a cycle. We have $\langle P\rangle=G$ and there are two possibilities. If

$$
P=f_{1} w_{1} f_{2} w_{2} \ldots f_{t-1} w_{t-1} f_{t}, \quad t \geq 2,
$$

where $f_{1} f_{t} \in E(G), f_{i} \in F, 1 \leq i \leq t$, and $w_{i} \in W, 1 \leq i \leq t-1$, then the set

$$
F^{\prime}=\left\{f_{2}, f_{4}, f_{6}, \ldots\right\}
$$

satisfies the necessary properties. If

$$
P=f_{1} w_{1} f_{2} w_{2} \ldots f_{t} w_{t}, \quad t \geq 2
$$

where $f_{1} w_{t} \in E(G), f_{i} \in F$ and $w_{i} \in W, 1 \leq i \leq t$, then $\langle P\rangle$ is an alternating cycle. Since $2 t=|P| \neq 4 k+2$, it follows that $t$ is even and the set

$$
F^{\prime}=\left\{f_{1}, f_{3}, \ldots, f_{t-1}\right\}
$$

gives the desired result.

Suppose now that the statement of Lemma 1 holds for any block-cactus graph having fewer vertices than $G$, and let $G$ consist of at least two blocks. Then there exists an end block $B$ of $G$ with only one cut vertex $v$ of $G$.

Case 1. The block $B$ is a complete graph.

Subcase 1.1. The cut vertex $v$ is an element of $F$. Assume that $V(B) \subseteq F$. If we consider the block-cactus graph $G^{\prime}=G-(V(B)-\{v\})$, then $\left|V\left(G^{\prime}\right)\right|<|V(G)|$, and $\left|N_{G^{\prime}}(w) \cap F\right| \geq 2$ for all $w \in V\left(G^{\prime}\right)-F$. Hence, by the induction hypothesis, the desired result easily follows.

Let $V(B) \cap W \neq \emptyset$. Now $G^{\prime}=G-\left(V(B) \cup\left(N_{G}(v) \cap W\right)\right) \neq \emptyset$ is a block-cactus graph such that $\left|N_{G^{\prime}}(w) \cap F\right| \geq 2$ for all $w \in V\left(G^{\prime}\right)-F$. Again, by the induction hypothesis we obtain the statement of the lemma.

Subcase 1.2. The cut vertex $v$ is an element of $W$. If $|F \cap V(B)| \geq 2$, then the block-cactus graph $G^{\prime}=G-V(B)$ together with the induction hypothesis (as well as using $v$ ) yields the desired result.

If $|F \cap V(B)| \leq 1$, then $|F \cap V(B)|=1$ so let $F \cap V(B)=\{b\}$. Since $|N(v) \cap F| \geq 2$, it follows that there exists a further neighbor $a \in F$ of $v$ in $G-V(B)$. Now we define $G^{\prime}=G-\left(V(B) \cup\{a\} \cup\left(N_{G}(a) \cap W\right)\right)$. If $G^{\prime}=\emptyset$, then $F^{\prime}=\{a\}$ fulfills the statement of Lemma 1. Finally, if $G^{\prime} \neq \emptyset$, then by the induction hypothesis there exists a set $F^{*} \subseteq F-\{a, b\}$ with $W \cap V\left(G^{\prime}\right) \subseteq N_{G^{\prime}}\left(F^{*}\right)$ and $2\left|F^{*}\right| \leq|F|-2$. Consequently, for $F^{\prime}=F^{*} \cup\{a\} \subseteq F$, we deduce that $W \subseteq N_{G}\left(F^{\prime}\right)$ and $2\left|F^{\prime}\right| \leq|F|$.

Case 2. The block $B$ is a cycle.

Subcase 2.1. Suppose that $v \in F$. If $N_{B}(v) \cap W=\emptyset$, then the graphs $B-\{v\}$ and $G-(V(B)-\{v\})$ together with the induction hypothesis yield the desired result. Therefore we can assume that there is $w_{1} \in N_{B}(v) \cap W$. Let $P^{\prime}$ be the maximal alternating path in the graph $B-\{v\}$ such that $w_{1} \in P^{\prime}$. Consider the path $P=P^{\prime} \cup\{v\}$. Suppose firstly that $P$ has the following form:

$$
P=v w_{1} f_{1} \ldots w_{t} f_{t}, \quad t \geq 1,
$$


where $f_{i} \in F$ and $w_{i} \in W, 1 \leq i \leq t$. The graph $\langle P\rangle$ is either a path or a cycle depending on the existence of the edge $v f_{t}$. If $t$ is even, then the set $\left\{f_{1}, f_{3}, \ldots, f_{t-1}\right\}$ dominates $P^{\prime} \cap W$ and the graph $G-P^{\prime}$ together with the induction hypothesis gives the desired result. If $t$ is odd, then the set $\left\{v, f_{2}, f_{4}, \ldots, f_{t-1}\right\}$ dominates the set $(P \cup N(v)) \cap W$. By the induction hypothesis, the statement of Lemma 1 holds for the graph $G-P-(N(v) \cap W)$, and the result easily follows.

Now suppose that

$$
P=v w_{1} f_{1} \ldots w_{t} f_{t} w_{t+1}, \quad t \geq 1,
$$

where $f_{i} \in F, 1 \leq i \leq t$, and $w_{i} \in W, 1 \leq i \leq t+1$. We have $v w_{t+1} \in E(G)$, i.e., $\langle P\rangle$ is an alternating cycle and $\langle P\rangle=B$. Now $2 t+2=|P| \neq 4 k+2$ and hence $t$ is odd. The set $\left\{v, f_{2}, f_{4}, \ldots, f_{t-1}\right\}$ dominates $(P \cup N(v)) \cap W$, and the graph $G-P-(N(v) \cap W)$ together with the induction hypothesis gives the desired result.

Subcase 2.2. Suppose that $v \in W$. The set $V(B) \cap W$ does not contain edges of $G$ and therefore $|N(v) \cap V(B) \cap F| \geq 2$. The graphs $B$ and $G-B$ satisfy the conditions of Lemma 1. By the induction hypothesis, there are corresponding dominating sets and the union of these sets yields the set $F^{\prime}$. The proof is complete.

\section{Main Result}

Let $\pi(G)$ denote the number of induced cycles of length $2(\bmod 4)$ in a graph $G$. The following theorem gives the ratio of the irredundance and domination numbers for blockcactus graphs in terms of $\pi(G)$. We will see later that this ratio is strict.

Theorem 1 If $G$ is a block-cactus graph, then

$$
\frac{i r(G)}{\gamma(G)} \geq \frac{5 \pi(G)+4}{8 \pi(G)+6}
$$

Proof. Let $I$ be an $i r$-set of $G$, i.e., $I$ is a maximal irredundant set and $|I|=i r(G)$, and denote $U=V(G)-N[I]$.

We say that $G$ contains an $S$-subgraph if there exist sets $\left\{v_{1}, v_{2}, v_{3}\right\} \subseteq I$ and $S=$ $\left\{u, v_{i}^{\prime}, v_{i}^{\prime \prime}, i=1,2,3\right\}$ satisfying the following conditions:

$$
P\left(v_{i}, I\right)=\left\{v_{i}^{\prime}, v_{i}^{\prime \prime}\right\}, i=1,2,3, \quad\left\{v_{1}^{\prime}, v_{1}^{\prime \prime}\right\} \subseteq N\left(v_{2}^{\prime}\right), \quad\left\{v_{3}^{\prime}, v_{3}^{\prime \prime}\right\} \subseteq N\left(v_{2}^{\prime \prime}\right),
$$

and

$$
\left\{v_{2}^{\prime}, v_{2}^{\prime \prime}\right\} \subseteq N(u)
$$

for $u \in U$. Note that the vertices $v_{1}, v_{2}, v_{3}$ are not isolated in the graph $\langle I\rangle$, since $v_{i} \notin$ $P\left(v_{i}, I\right)$ for $i=1,2,3$.

Now suppose that $G$ contains an $S$-subgraph. Remove from $G$ the vertices of $S$ together with incident edges, and add the set $S^{\prime}=\left\{w_{1}, w_{2}, p_{i}, u_{i}, i=1,2,3\right\}$ together with edges $w_{1} v_{1}, w_{1} v_{2}, w_{2} v_{2}, w_{2} v_{3}$ and $v_{i} p_{i}, p_{i} u_{i}, i=1,2,3$. Denote the resulting graph by $G^{\prime}$. The vertices $v_{1}, v_{2}, v_{3}$ belong to different connected components of the graph $G-S$, since otherwise $G$ is not a block-cactus graph. Therefore $G^{\prime}$ is a block-cactus graph and $G^{\prime}$ does not contain new cycles, i.e., $\pi\left(G^{\prime}\right) \leq \pi(G)$. Furthermore,

$$
P_{G^{\prime}}(x, I)=P_{G}(x, I) \neq \emptyset \quad \text { for each } \quad x \in I-\left\{v_{1}, v_{2}, v_{3}\right\}
$$


and

$$
P_{G^{\prime}}\left(v_{i}, I\right)=\left\{p_{i}\right\} \quad \text { for } \quad i=1,2,3 .
$$

Consequently, the set $I$ is irredundant in $G^{\prime}$. Let $z$ be a vertex of $V(G)-I-S$. The set $I \cup\{z\}$ is redundant in $G$, since $I$ is a maximal irredundant set in $G$. By definition, either

$$
N_{G}[z] \subseteq N_{G}[I]
$$

or

$$
P_{G}(y, I) \subseteq N_{G}[z]
$$

for some vertex $y \in I$. Note that $y \notin\left\{v_{1}, v_{2}, v_{3}\right\}$, since otherwise $G$ is not a block-cactus graph. Therefore $I \cup\{z\}$ is a redundant set in $G^{\prime}$. If $z \in S^{\prime}$, then it is straightforward to see that $I \cup\{z\}$ is a redundant set in $G^{\prime}$. Thus $I$ is a maximal irredundant set in $G^{\prime}$. Now let $D$ denote a minimum dominating set in $G^{\prime}$. We have $\left|D \cap\left(S^{\prime} \cup\left\{v_{1}, v_{2}, v_{3}\right\}\right)\right| \geq 4$ and the set $\left(D-\left(S^{\prime} \cup\left\{v_{1}, v_{2}, v_{3}\right\}\right)\right) \cup\left\{u, v_{1}, v_{2}, v_{3}\right\}$ is a dominating set of $G$. Hence $\gamma\left(G^{\prime}\right) \geq \gamma(G)$.

Applying the above construction to $G$ we can obtain a block-cactus graph $H$ such that $I$ is a maximal irredundant set in $H$ and $H$ does not contain $S$-subgraphs with respect to I. Moreover,

$$
\pi(H) \leq \pi(G) \text { and } \quad \gamma(H) \geq \gamma(G) .
$$

Consider now the graph $H$ and denote $U=V(H)-N[I]$. By Proposition 1, for any vertex $u \in U$ there is a vertex $f(u) \in I$ such that $P(f(u), I) \subseteq N(u)$. Put

$$
A=\{f(u): u \in U\} \cup\{v \in I:|P(v, I)|=1 \text { and } v \notin P(v, I)\} .
$$

Form the set $B$ choosing for each vertex $a \in A$ one vertex from $P(a, I)$ by the following rule. If $P(a, I)=\{p\}$, then we add $p$ into $B$. If $|P(a, I)|>1$, then there is a vertex $u \in U$ such that $P(a, I) \subseteq N(u)$. Since $H$ is a block-cactus graph, we have $P(a, I)=\left\{p_{1}, p_{2}\right\}$ and $p_{1} p_{2} \notin E(H)$. By Proposition $1, p_{i}$ dominates $P\left(y_{i}, I\right)$, where $y_{i} \in I-\{a\}, i=1,2$. It is evident that $y_{i} \notin P\left(y_{i}, I\right)$. We have $y_{1} \neq y_{2}$ and $\left|P\left(y_{i}, I\right)\right| \leq 2$, since otherwise $H$ is not a block-cactus graph. The graph $H$ has no $S$-subgraph, and so without loss of generality $\left|P\left(y_{1}, I\right)\right|=1$. Now add $p_{2}$ into $B$. Note that $y_{1} \in A$. Therefore $P\left(y_{1}, I\right) \in B$ and the vertex $p_{1}$ is dominated by $B$.

Thus the set $B$ dominates $A \cup U \cup\{P(a, I): a \in A\}$ and $|B|=|A|$. Let $C=(I-A) \cup B$ and let $C$ dominate $V(H)-W$. We have $|C|=|I|=\operatorname{ir}(G)$ and for each $w \in W$,

$$
\left|N_{H}(w) \cap A\right| \geq 2
$$

Denote

$$
D=\left\{u \in I: N_{\langle I\rangle}(u) \neq \emptyset\right\} .
$$

Clearly, for each vertex $d \in D$,

$$
\operatorname{deg}_{\langle D\rangle} d \geq 1
$$

By the definitions of $A$ and $D$,

$$
A \subseteq D \subseteq I
$$

and therefore for each $w \in W$, 


$$
\left|N_{H}(w) \cap D\right| \geq 2 .
$$

Suppose that the graph $\langle D \cup W\rangle$ has no D-W-alternating cycle of length $2(\bmod 4)$. Then, by Lemma 1 ,

$$
\gamma(G) \leq \gamma(H) \leq \frac{3}{2}|I|=\frac{3}{2} \operatorname{ir}(G)
$$

which implies the desired inequality. Define now the graph $F$ with the vertex set $D$ as follows. Replace in the graph $\langle D \cup W\rangle$ all alternating cycles $C^{1}, C^{2}, \ldots, C^{k}(k \geq 1)$ of length $2(\bmod 4)$ by complete graphs and denote the resulting graph by $H_{1}$. Let $F$ be the subgraph of $H_{1}$ induced by $D$. It is obvious that $H_{1}$ and $F$ are block-cactus graphs and the sets $K^{i}=C^{i} \cap D, 1 \leq i \leq k$, induce complete subgraphs in $F$. Moreover, the $K^{i}$ are blocks in $F$ and $\left|K^{i}\right| \geq 3$ for all $i=1,2, \ldots, k$. Call the blocks $K^{i}$ special.

We will add a set of extra edges in the set $D$ of the graph $H_{1}$ in such a way that the resulting graph $H^{*}$ possesses Property A.

Property A. For any vertex $w \in W$ in the graph $H^{*}$ there exist vertices $u, v \in N(w) \cap D$ such that either

$$
\operatorname{deg}_{\langle D\rangle} u \geq 2 \quad \text { and } \quad \operatorname{deg}_{\langle D\rangle} v \geq 2,
$$

or

$$
N_{\langle D\rangle}(u)=\{v\} .
$$

Construct the sequence of block-cactus graphs

$$
H_{1}, H_{2}, \ldots, H_{m}
$$

in accordance with the following rule. Suppose that we have the block-cactus graph $H_{i}$ and it contains the vertex $w_{i} \in W \cap V\left(H_{i}\right)$ and the vertices $u_{i}, v_{i} \in N_{H_{i}}\left(w_{i}\right) \cap D$ satisfying

$$
\operatorname{deg}_{\langle D\rangle} u_{i}=1 \text { and } u_{i} v_{i} \notin E\left(H_{i}\right) .
$$

If the vertices $u_{i}$ and $v_{i}$ belong to different connected components of the graph $H_{i}-\left\{w_{i}\right\}$, then

$$
H_{i+1}=\left(H_{i}-\left\{w_{i}\right\}\right) \cup u_{i} v_{i}
$$

is a block-cactus graph. If the vertices $u_{i}$ and $v_{i}$ belong to one connected component of the graph $H_{i}-\left\{w_{i}\right\}$, then the vertices $u_{i}, w_{i}, v_{i}$ in the graph $H_{i}$ belong to a block which is a cycle. Again, the graph $H_{i+1}$ is a block-cactus graph.

Thus, the graph $H_{m}$ is a block-cactus graph. Taking into account (2) and (3) we see that for any vertex $w \in W \cap V\left(H_{m}\right)$ there exist vertices $u, v \in N_{H_{m}}(w) \cap D$ such that either $\operatorname{deg}_{\langle D\rangle} u \geq 2$ and $\operatorname{deg}_{\langle D\rangle} v \geq 2$, or $N_{\langle D\rangle}(u)=\{v\}$. Moreover, in the graph $H_{m}$, $\operatorname{deg}_{\langle D\rangle} u_{i} \geq 2$ and $\operatorname{deg}_{\langle D\rangle} v_{i} \geq 2$ for all $i=1,2, \ldots, m-1$. Put

$$
F^{*}=F \cup_{i=1}^{m-1} u_{i} v_{i} \quad \text { and } \quad H^{*}=H_{1} \cup_{i=1}^{m-1} u_{i} v_{i} .
$$

The graph $F^{*}$ is a block-cactus graph, since it is an induced subgraph of $H_{m}$. Furthermore, $H^{*}-\cup_{i=1}^{m-1} w_{i}=H_{m}$, and therefore the graph $H^{*}$ satisfies Property A.

For the above alternating cycles $C^{i}$, the sets $C_{i} \cap D, i=1,2, . ., k$, do not contain edges in the graph $H$. By the definitions of the set $D$ and the graph $F^{*}$, we obtain the following property. 
Property B. For any vertex $u \in V\left(F^{*}\right)$ there is the edge $u v \in E\left(F^{*}\right)$ not belonging to any block $K^{i}, 1 \leq i \leq k$.

Let the graph $F^{*}$ contain $r \in\{0,1, \ldots, k\}$ special blocks $K^{i}$ satisfying Property C. Without loss of generality we may assume that the following blocks possess this property:

$$
K^{1}, K^{2}, \ldots, K^{r}
$$

Property C. The block $K^{i}$ contains the vertex $v_{i}$ such that

$$
N_{F^{*}}\left(v_{i}\right)-K^{i}=\left\{p_{i}\right\} \quad \text { and } \quad \operatorname{deg}_{F^{*}} p_{i}=1 .
$$

Lemma 2 For the graph $F^{*}$,

$$
\left|V\left(F^{*}\right)\right|=|D| \geq 5 k-3 r+4 .
$$

Proof. We prove (4) by induction on the number $k$ of special blocks. Let $k=1$. Taking into account Properties B and C, we obtain $\left|V\left(F^{*}\right)\right| \geq 3\left|K^{1}\right| \geq 9$ if $r=0$, and $\left|V\left(F^{*}\right)\right| \geq$ $2\left|K^{1}\right| \geq 6$ if $r=1$. Now suppose that (4) holds for any block-cactus graph having fewer special blocks $K^{i}$ with $\left|K^{i}\right| \geq 3$ and satisfying Property B. If $F^{*}$ is not a connected graph, then the result easily follows. Let $F^{*}$ be a connected graph and denote

$$
K=\cup_{i=1}^{k} K^{i}
$$

For the vertex $u \in K$ denote by $B_{u}$ all connected components of the graph $F^{*}-\{u\}$ which do not contain vertices of the set $K$. The graph

$$
F^{*}-\cup_{u \in K} B_{u}
$$

has an end block $K^{t}$ with only one cut vertex $x$. By Property $\mathrm{B}$, there is the edge $x y \in$ $E\left(F^{*}\right)$ such that $x y \notin K^{i}$ for any $i=1,2, \ldots, k$. Consider the graph

$$
F^{\prime}=F^{*}-\cup_{u \in K^{t}-\{x\}}\left(B_{u} \cup\{u\}\right) .
$$

It is evident that $F^{\prime}$ is a block cactus graph having $k-1$ special blocks and satisfying Property B. Suppose that some block $K^{i}, r<i \leq k$, satisfies Property C in the graph $F^{\prime}$. The first possibility is that $\operatorname{deg}_{F^{\prime}} x=1$ and $y \in K^{i}$. The second possibility is that $\operatorname{deg}_{F^{\prime}} y=1$ and $x \in K^{i}$. In either of these two cases we add to $F^{\prime}$ the new vertex $z$ and the edge $x z$. The block $K^{i}$ does not satisfy Property $\mathrm{C}$ in the resulting graph, and this operation evidently does not produce new special blocks satisfying Property C. Thus, if $i \in\{r+1, r+2, \ldots, k\}$, then the block $K^{i}$ does not satisfy Property $\mathrm{C}$ in the graph $F^{\prime}$ and

$$
V\left(F^{*}\right)=\cup_{u \in K^{t}-\{x\}}\left(B_{u} \cup\{u\}\right) \cup V\left(F^{\prime}\right)-\{z\} .
$$

Case 1. If $t \leq r$, then $F^{\prime}$ contains exactly $r-1$ special blocks satisfying Property C. Using the induction hypothesis, Property B and the inequality $\left|K^{t}\right| \geq 3$, we see that

$$
\begin{aligned}
\left|V\left(F^{*}\right)\right| & \geq\left|\cup_{u \in K^{t}-\{x\}} B_{u} \cup\{u\}\right|+\left|V\left(F^{\prime}\right)\right|-1 \\
& \geq 2\left(\left|K^{t}\right|-1\right)+5(k-1)-3(r-1)+3 \\
& \geq 5 k-3 r+5 .
\end{aligned}
$$


Case 2. If $t>r$, then $F^{\prime}$ contains exactly $r$ special blocks satisfying Property C. The block $K^{t}$ does not satisfy Property $\mathrm{C}$ and hence $\left|B_{u}\right| \geq 2$ for each $u \in K-\{x\}$. We obtain

$$
\begin{aligned}
\left|V\left(F^{*}\right)\right| & \geq\left|\cup_{u \in K^{t}-\{x\}} B_{u} \cup\{u\}\right|+\left|V\left(F^{\prime}\right)\right|-1 \\
& \geq 3\left(\left|K^{t}\right|-1\right)+5(k-1)-3 r+3 \\
& \geq 5 k-3 r+4
\end{aligned}
$$

The proof of Lemma 2 is complete.

Now consider the sets

$$
V=\left\{v_{1}, v_{2}, \ldots, v_{k}\right\}
$$

and

$$
P=\left\{p_{1}, p_{2}, \ldots, p_{r}\right\},
$$

where $v_{i}$ and $p_{i}$ are the vertices defined in Property $\mathrm{C}$ if $i \leq r$, and $v_{i}$ is some vertex of $K^{i}$ if $i>r$. We have, $v_{i} p_{i} \in E\left(F^{*}\right)$ and $\operatorname{deg}_{F^{*}} p_{i}=1$ for $i=1,2, \ldots, r$. Note that the set $\left\{v_{1}, \ldots, v_{r}\right\}$ contains different vertices by Property $\mathrm{C}$, while the set $\left\{v_{r+1}, \ldots, v_{k}\right\}$ does not necessarily contain different vertices. Therefore,

$$
|V|-|P|=\left|\left\{v_{r+1}, \ldots, v_{k}\right\}\right| \leq k-r .
$$

Denote

$$
X=D-(V \cup P)
$$

Lemma 3 For each vertex $w \in W-N_{H}(V)$ in the graph $H$,

$$
\left|N_{H}(w) \cap X\right| \geq 2 \text {. }
$$

Proof. Denote by $H^{\prime}$ the induced subgraph $\langle D \cup W\rangle$ in the graph $H$. By definitions, the graph $H^{*}$ is obtained from $H^{\prime}$ by adding edges in the sets $C^{1}, C^{2}, \ldots, C^{k}$ and the set $D$. Therefore, $N_{H^{\prime}}(w) \subset N_{H^{*}}(w)$ if $w \in C^{i} \cap W$, and $N_{H^{\prime}}(w)=N_{H^{*}}(w)$ if $w \in W-\cup_{i=1}^{k} C^{i}$. Now assume that $w \in W-N_{H^{\prime}}(V)$ and

$$
\left|N_{H^{\prime}}(w) \cap X\right| \leq 1 .
$$

Consider the case $w \in C^{i} \cap W$ where $i \in\{1,2, \ldots, k\}$. Since $C^{i}$ is an alternating cycle and $w \notin N_{H^{\prime}}(V)$, it follows that there are vertices $c^{\prime}, c^{\prime \prime} \in N_{H^{\prime}}(w) \cap C^{i} \cap D$ and $c^{\prime}, c^{\prime \prime} \notin V$. In the graph $H^{*}$ we have $c^{\prime}, c^{\prime \prime} \in K^{i}$ and therefore $c^{\prime}, c^{\prime \prime} \notin P$. Thus, $c^{\prime}, c^{\prime \prime} \in X$ and $\left|N_{H^{\prime}}(w) \cap X\right| \geq 2$, a contradiction. Now consider the case $w \in W-\cup_{i=1}^{k} C^{i}$. Since $N_{H^{\prime}}(w)=N_{H^{*}}(w)$, we have $N_{H^{*}}(w) \cap V=\emptyset$. Thus, in the graph $H^{*}$ the vertex $w$ is adjacent only to vertices of $P$ and to at most one vertex of $X$, contrary to Property A. The proof of Lemma 3 is complete.

In the graph $H$ consider the induced subgraph $X \cup W^{\prime}$, where $W^{\prime}=W-N_{H}(V)$. This graph is a block-cactus graph having no alternating cycles of length $2(\bmod 4)$ as induced subgraphs. By Lemma 3, $\left|N_{\left\langle X \cup W^{\prime}\right\rangle}(w) \cap X\right| \geq 2$ for each vertex $w \in W^{\prime}$. By Lemma 1 , there exists $X^{\prime} \subseteq X$ such that $X^{\prime}$ dominates $W^{\prime}$ and $2\left|X^{\prime}\right| \leq|X|$. Thus, the set $T=V \cup X^{\prime}$ 
dominates $W$ in the graph $H$, and $C \cup T$ is a dominating set of $H$. Using (4), (5), and the inequality $k \geq r \geq 0$, we obtain

$$
\begin{gathered}
|T|=|V|+\left|X^{\prime}\right| \leq|V|+\frac{1}{2}(|D|-|V|-|P|)=\frac{1}{2}(|D|+|V|-|P|) \\
\leq \frac{|D|+k-r}{2|D|}|I| \leq \frac{3 k-2 r+2}{5 k-3 r+4}|I| \leq \frac{3 k+2}{5 k+4}|I| .
\end{gathered}
$$

Using (1) and the inequality $k \leq \pi(H)$, we finish the proof of Theorem 1

$$
\gamma(G) \leq \gamma(H) \leq|C|+|T| \leq \frac{8 k+6}{5 k+4}|I| \leq \frac{8 \pi(G)+6}{5 \pi(G)+4} i r(G) .
$$

The following corollaries follow directly from Theorem 1.

Corollary 1 If $G$ is a block-cactus graph, then $\operatorname{ir}(G) / \gamma(G)>5 / 8$.

Since any cactus graph is a block-cactus graph, Corollary 1 proves Conjecture 1 . The example below shows that the bound $5 / 8$ is best possible for cactus graphs and, consequently, for block-cactus graphs.

Corollary 2 (Volkmann [11]) If $G$ is a block graph, then $\operatorname{ir}(G) / \gamma(G) \geq 2 / 3$.

The bound $2 / 3$ is best possible for block graphs (see [11]).

In conclusion we show that the bounds in Theorem 1 and Corollary 1 are sharp. Let $C^{i}=a_{i} b_{i} c_{i} d_{i} e_{i} f_{i} a_{i}, i=1,2, \ldots, k$ be simple cycles of length 6 and let $T^{i}=x_{i} y_{i} z_{i}, i=$ $0,1, \ldots, k+1$ be cycles of length 3 . Add the edges

$$
\left\{e_{i} a_{i+1}: 1 \leq i \leq k-1\right\}, \quad\left\{c_{i} x_{i}: 1 \leq i \leq k\right\}, \quad \text { and } \quad\left\{x_{0} a_{1}, e_{k} x_{k+1}\right\} .
$$

Put

$$
I=\left\{a_{i}, c_{i}, e_{i}: 1 \leq i \leq k\right\} \cup\left\{x_{i}, y_{i}: 0 \leq i \leq k+1\right\} .
$$

Also add the paths $P_{u}=u u^{\prime} u^{\prime \prime}$ for each vertex $u \in I$. Denote the resulting graph by $G$. The graph $G$ is both a block-cactus graph and a cactus graph.

Every maximal irredundant set of the graph $G$ contains at least one vertex of the set $\left\{u, u^{\prime}, u^{\prime \prime}: u \in I\right\}$. Therefore, $\operatorname{ir}(G) \geq|I|=5 k+4$. On the other hand, $I$ is a maximal irredundant set of $G$ and hence $\operatorname{ir}(G)=5 k+4$. It is not difficult to see that

$$
\left\{u^{\prime}: u^{\prime} \in P_{u}, u \in I\right\} \cup\left\{a_{i}, c_{i}: 1 \leq i \leq k\right\} \cup\left\{x_{i}: 0 \leq i \leq k+1\right\}
$$

is a minimum dominating set and therefore $\gamma(G)=8 k+6$. Thus,

$$
\operatorname{ir}(G) / \gamma(G)=(5 k+4) /(8 k+6) \quad \text { and } \quad \lim _{k \rightarrow \infty}(5 k+4) /(8 k+6)=5 / 8
$$

Acknowledgment The author thanks the referees for their helpful suggestions. 


\section{References}

[1] R. B. Allan and R. Laskar, On domination and some related concepts in graph theory, Proc. 9th Southeast Conf. on Combin., Graph Theory and Comp. (Utilitas Math., Winnipeg, 1978) 43-56.

[2] B. Bollobás and E. J. Cockayne, Graph-theoretic parameters concerning domination, independence, and irredundance, J. Graph Theory 3 (1979) 241-249.

[3] E. J. Cockayne and S. T. Hedetniemi, Independence graphs, Proc. 5th Southeast Conf. on Combin., Graph Theory and Comp. (Utilitas Math., Winnipeg, 1974) 471-491.

[4] P. Damaschke, Irredundance number versus domination number, Discrete Math. 89 (1991) 101-104.

[5] O. Favaron and D. Kratsch, Ratios of domination parameters, Advances in Graph Theory, ed. V. R. Kulli (Vishwa Intern. Publications, 1991) 173-182.

[6] Y. Guo and L. Volkmann, A generalization of Menger's theorem for certain blockcactus graphs, Graphs and Combin. 11 (1995) 49-52.

[7] J. H. Hattingh and M. A. Henning, The ratio of the distance irredundance and domination numbers of a graph, J. Graph Theory 18 (1994) 1-9.

[8] B. Randerath and L. Volkmann, A characterization of well covered block-cactus graphs, Australasian J. Combin. 9 (1994) 307-314.

[9] D. Rautenbach and L. Volkmann, The domatic number of block-cactus graphs, Discrete Math. (to appear)

[10] L. Volkmann, A reduction principle concerning minimum dominating sets in graphs, Preprint, Lehrstuhl II für Mathematik, RWTH Aachen, 1991.

[11] L. Volkmann, The ratio of the irredundance and domination number of a graph, Discrete Math. 178 (1998) 221-228. 